\title{
Konstitution und Dauer sozialer Kontinuanten
}

\section{Soziale Entitäten}

Was haben Fußballmannschaften, Bundestagssitzungen, Landesgrenzen und Zwanzig-Euro-Scheine gemeinsam? Sie sind alle soziale Entitäten, d.h., mit metaphysischer Notwendigkeit verdanken sie ihre Existenz sozialem Handeln, d.h. einem Handeln von zwei oder mehr Handelnden, die sich in ihrem Denken und Handeln auf das Denken und Handeln des oder der anderen beziehen. ${ }^{1}$ Einige, aber nicht alle sozialen Entitäten sind Kollektive, d.h., sie haben Mitglieder. Fußballmannschaften haben Mitglieder, ebenso wie Familien und Orchester. Alle sozialen Entitäten sind aber Institutionen, d.h. durch bestimmte soziale Handlungen etabliert worden: Fußballmannschaften werden gegründet, Bundestagssitzungen werden einberaumt und eröffnet, Landesgrenzen werden gezogen und verteidigt, Zwanzig-Euro-Scheine werden von offizieller Stelle herausgegeben. Institutionen sind stets in menschliches Handeln eingebettet, aber manche Institutionen umfassen selber keine Menschen, sind also keine Kollektive, wie die Institution des Geldes, eine politische Grenze, sprachliche Äußerungen oder Verträge. Auch Bundestagssitzungen haben zwar Beteiligte, aber keine Mitglieder. Die Beispiele zeigen, dass soziale Entitäten zu ganz unterschiedlichen ontologischen Kategorien gehören können. Es gibt soziale Sachverhalte oder Tatsachen, aber auch soziale Ereignisse und soziale Akte, soziale Rollen, soziale Qualitäten, soziale Quantitäten, soziale Relationen und auch soziale Substanzen, wie etwa juristische Personen. ${ }^{2}$

Warum gibt es überhaupt soziale Entitäten? Mit einer solchen Frage

${ }^{1}$ Diese Charakterisierung sozialen Handelns orientiert sich an dem Vorschlag von Max Weber; vgl. Weber (1921), § 1/II, 11 und (1913), S. 441 (dort zur Erläuterung von „Gemeinschaftshandeln“).

${ }^{2}$ Vgl. dazu ausführlicher Jansen (2005). 
können wir nach der causa fiendi, der Ursache der Entstehung eines Dinges, fragen; aber die Frage kann auch auf die causa essendi zielen, also auf den „Seinsgrund“ eines Dinges, der nicht die Genese, sondern die (fortgesetzte) Existenz des Dinges erklärt. ${ }^{3}$ Fragen wir nach seinem „Seinsgrund“, dann können wir unsere Frage durch die Angabe eines Konstitutionsmodells beantworten. In einem Konstitutionsmodell wird Sein und Existenz eines konstituierten Dinges zurückgeführt auf Sein und Existenz seiner Konstituenten. Konstituierte Entitäten existieren also, weil ihre Konstituenten existieren, ${ }^{4}$ unabhängig davon, wie sie entstanden sind (und auch unabhängig davon, ob sie überhaupt entstanden sind).

Für soziale Entitäten sind ganz unterschiedliche Analysen vorgeschlagen worden, die wir für die Entwicklung von ontologischen Konstitutionsmodellen verwenden können. Margaret Gilbert etwa lädt uns ein, die Konstitution von Wir-Gruppen als das Eingehen einer, ,gemeinsamen Verpflichtung“ (joint commitment) durch die beteiligten Gruppenmitglieder zu verstehen. ${ }^{5}$ John Searle hingegen schlägt vor, die Konstitution sozialer Entitäten als das Übertragen eines sozialen Status auf ein natürliches Objekt (oder ein anderes soziales Objekt) mit Hilfe einer konstitutiven Regel der Form „X zählt als Y im Kontext K“ zu analysieren. ${ }^{6}$ Andere Autoren sehen die symbolischen Repräsentationen sozialer Entitäten, ihre „Inskriptionen“, als konstitutiv für die Entitäten $a^{7}$ oder aber die durch kommissive Sprechakte (oder auch „Dokumentenakte ${ }^{\text {(68) }}$ ) wie Versprechen und Verträge entstandenen deontischen Strukturen von Verpflichtungen und Rechten. ${ }^{9}$

Die im Folgenden zugrunde gelegte Arbeitshypothese wird sein, dass

\footnotetext{
${ }^{3}$ Für diesen Kontrast vgl. bereits Aristoteles, Metaphysik V 1, 1013a18-19.

${ }^{4}$ Dies ist die Kernthese einer explanatorischen Analyse der Konstitutionsrelation, wie sie z.B. von Doepke (1996), Kap. 7 verteidigt wird. Für eine Kritik vgl. Wasserman (2004), S. 699-701.

${ }^{5}$ Vgl. v. a. Gilbert (1989). Für Gilbert selbst steht die ontologische Analyse nicht im Vordergrund; sie thematisiert aber immer wieder auch ontologische Fragen.

${ }^{6}$ Vgl. Searle $(1995)=(1997)$. Inzwischen hat Searle seine Theorie modifiziert und erweitert; vgl. Searle (2010).

${ }^{7}$ So z.B. Ferraris (2005) v. a. im Anschluß an Derrida.

${ }^{8}$ Ich übernehme diesen Ausdruck von Barry Smith, der in verschiedenen Vorträgen Ansätze zu einer der Sprechakt-Theorie analogen Theorie der „document acts“ vorgestellt hat.

${ }^{9}$ Vgl. Reinach (1913); zur Klassifikation von Sprechakten vgl. Searle (1969), Kap. 1.
} 
Konstitutionsmodelle (oder zumindest gute Konstitutionsmodelle) nicht nur eine Erklärung für die Existenz eines Dinges liefern, sondern auch für die Persistenz eines Dinges. Denn wenn gilt, dass ein Ding existiert, weil seine Konstituenten existieren, dann gilt auch, dass es nur dann existiert, wenn es Dinge gibt, die es konstituieren. Dann aber gilt auch, dass es genau so lange existiert, wie es Konstituenten gibt, deren Existenz für die Existenz des Dinges sowohl je einzeln notwendig als auch gemeinsam hinreichend ist.

Dieses Prinzip funktioniert einwandfrei, wenn ein Ding rigide von seinen Konstituenten abhängt, d.h., wenn es nicht fortexistieren kann, ohne dass seine Konstituenten fortexistieren. ${ }^{10}$ Lockes klassischer Analyse zufolge ist dies bei Massestücken der Fall, die Locke als eine Ansammlung von bestimmten Atomen beschreibt, so dass schon der Verlust eines einzigen solchen Atoms dazu führt, dass dieses Materiestück aufhört zu existieren. ${ }^{11}$ Wenn also, wie hier, ein Ding schon mit dem Verlust einer einzelnen Konstituente aufhört zu existieren, können wir noch einen Schritt weitergehen und sogar sagen, dass ein solches konstituiertes Ding nicht nur existiert, weil seine Konstituenten existieren, sondern auch persistiert, weil seine Konstituenten persistieren.

Die Sache wird dadurch kompliziert, dass viele Entitäten nicht rigide von ihren Konstituenten abhängen, sondern nur generisch. Wir selbst geben dafür ein gutes Beispiel ab. Können wir doch den vollständigen Austausch aller Atome unseres Körpers überleben, wenn dieser nur langsam genug vonstatten geht. Im aristotelischen Hylomorphismus gibt es nun neben der Materie, von der unsere Existenz generisch abhängt, noch die Form, die diese (wechselnde) Materie realisiert, die über die ganze Dauer des Individuums numerisch dieselbe bleibt. Anders als ein Massestück persistiert ein Mensch dann gerade nicht, weil seine Materie dieselbe bleibt; das könnte bei einem stoffwechselnden Wesen sogar ziemlich schnell zum Ende seiner Existenz führen. Ein Mensch persistiert im hylomorphistischen Bild vielmehr, weil seine Form dieselbe bleibt. Ein Mensch ist von beiden seiner hylomorphistischen Konstituenten Form und Materie ontologisch abhängig, aber nur von der Form ist er in rigider Wei-

\footnotetext{
${ }^{10}$ Für einen Überblick über Varianten ontologischer Abhängigkeit vgl. Lowe (2008).

${ }^{11}$ Vgl. Locke, Essay, Buch II, Kap. 27, § 3.
} 
se abhängig. Sowohl Form, als auch Materie sind Seinsursachen des Menschen, aber seine Persistenz hängt in diesem Fall nur von der Seinsursache Form ab, von der er in rigider Weise abhängig ist.

Mit Blick auf solche Konstituenten, von denen eine rigide Abhängigkeit besteht, können wir dann aber wieder sagen, dass ein Ding persistiert, solange und weil diese Konstituenten persistieren; mit Blick auf solche Konstituenten, von denen eine generische Abhängigkeit besteht, müssen wir jedoch sagen, dass ein Ding persistiert, solange und weil Konstituenten dieser Art existieren.

\section{Die zeitlichen Karrieren von sozialen Kontinuanten}

\subsection{Kontinuanten und Okkurrenten}

Soziale Entitäten haben in der Regel zeitliche Karrieren. Die zeitlichen Karrieren von Entitäten können von zweierlei Art sein. Entweder bestehen sie darin, dass sich eine Entität so über die Zeit erstreckt, dass sie zu keinem Zeitpunkt ganz vorhanden ist. Von dieser Art sind Ereignisse, Prozesse und Zeitintervalle, die aufgrund dieser Gemeinsamkeit als „Okkurrenten" bezeichnet werden. Okkurrenten haben einen zeitlichen Anfang und ein zeitliches Ende und erstrecken sich zwischen ihnen. Ein Fußballspiel beginnt beispielsweise mit einem Anpfiff, dauert zwei Halbzeiten lang, zwischen denen eine Pause liegt, und endet dann mit dem Schlusspfiff, sofern nicht eine Verlängerung oder ein Elfmeterschießen nötig wird.

Eine Entität kann aber auch zu verschiedenen aufeinanderfolgenden Zeitpunkten existieren und dabei zu jedem dieser Zeitpunkte als Ganzes vorliegen: Der Schiedsrichter etwa existiert mit all seinen - räumlichen Teilen sowohl beim Anpfiff als auch beim Abpfiff des Spieles. Dinge, für die dies gilt, werden „Kontinuanten“ genannt; sie sind nach der klassischen Definition von Johnson etwas, ,which continues to exist while its states or relations may be changing." ${ }^{212}$ Auch Kontinuanten haben eine zeitliche

12 Johnson (1921), S. 199. Zum Problem bloß instantan existierender Qualitäten etc. vgl. Johansson (2005); zum Ganzen vgl. auch Jansen (2008). Lewis (1986), S. 202 unterscheidet ganz ähnlich zwischen ,perdurers“ und ,,endurers“: „Something perdures iff it persists by having different temporal parts, or stages, at different times, though no one part of it is wholly present at more than one time; whereas it endures iff it persists by being wholly present at more than one time." 
Karriere: Sie entstehen, persistieren und hören auch wieder auf zu existieren. Die zeitliche Karriere eines Lebewesens nennt man üblicherweise sein „Leben“; bei sozialen Kontinuanten wie einem Staat oder einer Ehe könnte man von ihrer „Geschichte“ sprechen. Während ein Lebewesen, ein Staat oder eine Ehe selbst Kontinuanten sind, sind ihr Leben bzw. ihre Geschichte Okkurrenten. Mein Augenmerk wird im Folgenden vor allem den sozialen Kontinuanten gelten, und dadurch die Okkurrenten zwangsläufig vernachlässigen, die ich vor allem als Karrieren von Kontinuanten in den Blick nehme. Nacheinander werde ich zunächst die einzelnen Aspekten der zeitlichen Karriere der Kontinuanten im Detail diskutieren: ihre Existenz ( $\S$ 2.2), ihr Entstehen ( $\$ 2.3$ ) und ihr Vergehen ( 2.4$)$. Im nächsten Abschnitt werde ich dann verschiedene Theorien zur Persistenz sozialer Kontinuanten diskutieren $(\S 3)$.

\subsection{Die Existenz sozialer Kontinuanten}

So umstritten die Existenz sozialer Entitäten in manchen philosophischen Kreisen sein mag, im Alltagsleben ist sie evident: Tagtäglich gehen wir mit Kollektiven und Institutionen um. Beispiele für die Relevanz sozialer Kontinuanten im Alltagsleben gibt es zuhauf: Wir handeln in, mit und vielleicht auch gegen kollektive und kooperative Akteure, wir beachten Verkehrszeichen und folgen den Anweisungen des Polizisten, wir kaufen im Supermarkt ein und bezahlen die verlangten Preise, benutzen dafür Bargeld oder Kreditkarten als Zahlungsmittel. Ohne die Existenz von Verkehrsschildern und Polizisten würde der Straßenverkehr nicht so reibungslos funktionieren, und ohne Zahlungsmittel wie Bargeld und Kreditkarten sähe unser Wirtschafts- und Alltagsleben deutlich anders aus. Auch die Existenz politischer Grenzen, so willkürlich sie im Einzelfall gezogen sein mögen, ${ }^{13}$ hat enorme Auswirkungen auf unser Leben. Einerseits verbinden solche Grenzen die Menschen, die in ihnen leben, dadurch, dass sie den gleichen Gesetzen unterworfen sind und ihre Steuern und Abgaben an die gleichen Einrichtungen zahlen; andererseits trennen politische Grenzen die in ihnen lebenden Menschen von den Menschen, die jenseits der Grenzen leben und machen sie in leider nicht ganz seltenen Fällen sogar zu Feinden. Nicht zuletzt ist die Existenz der Staaten, um deren Grenzen es sich handelt, ein

${ }^{13}$ Vgl. Smith (1995). 
entscheidender Faktor in unserem sozialen Leben. Die Existenz (und Tätigkeit) gesetzgebender Kollektive in diesen Staaten sorgt wiederum für die Existenz gesetzlicher Regelungen; die Institutionen der Exekutive und der Jurisdiktion achten auf die Einhaltung dieser Regelungen bzw. sanktionieren die Verstöße gegen sie. Auch diese Entitäten - Finanzbeamte, Polizisten und Richter bzw. Finanzämter, Polizeireviere und Gerichte - existieren und haben einen Einfluss auf unser Leben.

Wenn soziale Entitäten nun aber einen Einfluss auf unser Leben haben, dann sind sie kausal relevant. Was aber kausal relevant ist, das existiert, denn kausale Relevanz kann nur Existierendes haben. Darüber hinaus benötigen wir soziale Entitäten, um das Geschehen in unserer Lebenswelt erklären und verstehen zu können. Weil soziale Entitäten desselben Typs aber ganz unterschiedliche physikalische „Realisierungen“ haben können, kommen soziale Entitäten in solchen Erklärungen in nicht reduzierbarer Form vor. Auch dies spricht dafür, dass wir besser davon ausgehen, dass soziale Entitäten tatsächlich existieren. Würden entgegen allem Anschein soziale Entitäten nicht existieren, wären weite Bereiche unserer Alltagssprache, aber auch die Wissenschaftssprache etwa der Juristen oder Politologen völlig anders zu interpretieren, als wir sie normalerweise verstehen. Das anzunehmen wäre aber eine sehr starke Einschränkung unserer linguistischen, aber auch unserer epistemischen Kompetenz: Sehr viele Existenzaussagen, die wir aufgrund unserer Alltagserfahrung für wahr halten, wären dann nämlich falsch, und wir könnten vielleicht gar nicht wissen, was es ,,wirklich“" gibt. ${ }^{14}$

\subsection{Das Entstehen sozialer Kontinuanten}

Dass soziale Entitäten entstehen bzw. entstanden sein müssen, gilt sowohl für jede einzelne soziale Entität als auch (und das ist die stärkere These) für soziale Entitäten in ihrer Gesamtheit. Letzteres ist augenscheinlich, wenn wir uns übliche naturgeschichtliche Vorstellungen vor Augen führen: In der „Kindheit“ des Universums, in der sich zunächst Galaxien und Planeten bilden müssen, gab es kein Leben, weder individuelles noch soziales, und somit auch keine sozialen Entitäten. Nun geht die moderne Naturwissenschaft zwar mit guten Gründen davon aus, dass es weder die biologi-

\footnotetext{
${ }^{14}$ Vgl. Baker (2007), S. 7.
} 
schen Arten, noch auch unser Universum immer gegeben hat, sondern dass beides irgendwann einmal entstanden ist. Aber eine metaphysische Notwendigkeit ist dies keineswegs. Wir können uns durchaus ein ,aristotelisches" Universum als eine mögliche Welt vorstellen, in der es immer schon Menschen gegeben hat und diese Menschen stets soziale Wesen waren. ${ }^{15}$ In diesem aristotelischen Universum hat es dann nie eine Zeit ohne soziale Entitäten gegeben. Das zeigt, dass es keineswegs notwendig ist, dass die sozialen Entitäten in ihrer Gesamtheit einmal entstanden sein müssen. Die Entstehung der sozialen Entitäten in ihrer Gesamtheit ist also keine metaphysische Notwendigkeit, sondern nur ein kontingenter Charakterzug unserer tatsächlichen Welt.

Aber auch in einem aristotelischen Universum können soziale Entitäten als Einzelne regelmäßig neu entstehen: Staaten können gegründet, Verfassungen neu entworfen werden. ${ }^{16}$ Und auch, wenn es im aristotelischen Universum, das ja keinen Anfang in der Zeit hat, niemals eine Zeit ohne Staaten oder Ehen gegeben haben sollte, ist es doch möglich, dass jeder Staat einmal gegründet und jede Ehe irgendwann einmal geschlossen wurde. Jeder Staat, jede Ehe und allgemein jede soziale Entität kann somit auch im aristotelischen Universum einen Anfang in der Zeit haben.

\subsection{Das Vergehen sozialer Kontinuanten}

Soziale Entitäten haben aber nicht nur einen Anfang in der Zeit, sondern auch ein zeitliches Ende: Ehen werden geschieden, Präsidenten werden abgewählt und Geldscheine werden entwertet. Selbst Staaten hören auf zu existieren, sei es durch Revolution oder durch Okkupation. Prominente Beispiele für das Ende von Staaten in der jüngsten Vergangenheit sind das Ende der UdSSR, der Union der sozialistischen Sowjetrepubliken, durch die Auflösung in mehrere Einzelstaaten, und das Ende der DDR, der Deutschen Demokratischen Republik, durch die Wiedervereinigung mit dem anderen deutschen Staat, der Bundesrepublik Deutschland. Auf dieses Bei-

\footnotetext{
15 Eine solche Welt setzt etwa die Biologie des Aristoteles voraus; vgl. z.B. Jansen 2010. Die Annahme, es habe immer Menschen gegeben, impliziert übrigens nicht, dass es immer Menschen geben muss: Durch atomare Verwüstung der Erde oder durch eine kollektive Fortpflanzungsverweigerung würde die Existenz der Menschheit recht schnell beendet werden.

${ }^{16}$ Vgl. Aristoteles, Politik III 3.
} 
spiel werde ich später noch einmal ausführlich zurückkommen.

\section{Die Persistenz sozialer Kontinuanten}

Wenn nun soziale Kontinuanten an einem bestimmten Zeitpunkt entstehen und zu einem anderen, späteren Zeitpunkt vergehen, dann müssen sie zwischen diesen beiden Zeitpunkten persistieren. Die Persistenz sozialer Kontinuanten ist somit ein direktes Korollar ihres Entstehens und späteren Vergehens. $^{17}$

Die in den letzen Jahrzehnten einflussreichsten Autoren für die ontologische Analyse der sozialen Welt sind wohl John Searle und Margaret Gilbert. Wenig überraschend äußern sich beide auch zur Persistenz von sozialen Entitäten. Ich werde nun darstellen, was John Searle und Margaret Gilbert zur Persistenz sozialer Kontinuanten sagen, und beide Ansätze miteinander vergleichen.

\subsection{Searle über Grenzen, Staaten und Ehen}

In seinem Buch über Die soziale Konstruktion der gesellschaftlichen Wirklichkeit beschreibt Searle institutionelle Tatsachen (institutional facts) als persistierende Entitäten. Institutionelle Tatsachen, so sagt er, „dauern in der Zeit, unabhängig von der Dauer der Bedürfnisse und Neigungen der Teilnehmer an der Institution." ${ }^{\text {18 }}$ Leo Zaibert hat mit einigem Recht darauf hingewiesen, dass Searle diese fortdauernde Existenz sozialer Entitäten intensiver diskutiert als die im Titel als Thema angekündigte „Konstruktion" sozialer Entitäten. ${ }^{19}$ Ich werde zwei unterschiedliche Ansätze herausarbeiten, die Searle zum Problem der Dauer sozialer Kontinuanten vorträgt. Searle unterscheidet diese beiden Ansätze nicht explizit. Ihnen ist gemeinsam, dass die Sprache eine zentrale Rolle für die Fortdauer sozialer Kontinuanten spielt:

„Diese fortdauernde Existenz erfordert ein Mittel der Repräsentation der Tatsachen, das unabhängig von den ursprünglichen vorsprachlichen psychologischen

\footnotetext{
${ }^{17}$ Das schließt eine eventuell lückenhafte Existenz sozialer Kontinuanten nicht aus; es folgt lediglich, dass soziale Kontinuanten überhaupt persistieren, nicht aber, dass sie lückenlos persistieren. Die Möglichkeit von Existenzlücken sozialer Kontinuanten diskutiere ich in Jansen (2007), S. 64-65.

${ }^{18}$ Searle (1997), S. $88=(1995)$, S. 78.

${ }^{19} \mathrm{Vgl}$. Zaibert (1998), S. 231.
} 
Zuständen der Teilnehmer ist, und solche Repräsentationen sind sprachlich.“ $(\text { Searle 1997, S.88 }=1995, \text { S.78 })^{20}$

Searle erläutert dies mit einer kleinen Geschichte: Eine Gruppe baut eine Mauer, um die Grenze ihres Gebietes zu markieren und nach außen zu verteidigen. Die Mauer weist physische Merkmale auf, die das Überschreiten der Grenze erschweren oder sogar unmöglich machen. Der Wall wird von der Gruppe aber nicht hinreichend ausgebessert und verfällt zusehends:

\begin{abstract}
„Man stelle sich vor, daß die Mauer allmählich zerfällt, daß das einzige, was übrigbleibt, eine Reihe von Steinen ist. Aber man stelle sich vor, daß die Einwohner und ihre Nachbarn die Steinreihe weiterhin als Grenze des Territoriums anerkennen, und zwar so, daß sie ihr Verhalten beeinflusst. Zum Beispiel überqueren die Einwohner die Grenze nur unter bestimmten Bedingungen, und $\mathrm{Au}-$ Benseiter können nur ins Territorium hineinwechseln, wenn es den Einwohnern akzeptabel erscheint. Die Reihe von Steinen hat jetzt eine Funktion, die nicht auf der Basis bloßer Physik erfüllt wird, sondern dank kollektiver Intentionalität." (Searle 1997, S.49=1995, S.39) ${ }^{21}$
\end{abstract}

Auch wenn der Wall nun keine physischen Merkmale aufweist, die das Überschreiten verhindern, kann er doch weiterhin als Grenze gelten, und es kann weiterhin akzeptiert werden, dass diese Grenze nicht überschritten werden darf. Die Grenze kann mithin unabhängig davon persistieren, ob es dort, wo sie verläuft, physische Hindernisse für ein Überschreiten gibt oder nicht.

Ein zweites Beispiel, das Searle diskutiert, ist der Zusammenbruch der kommunistischen Staaten in Europa im Jahr 1989. Searles Analyse der historischen Situation zufolge brach der Staatskommunismus zusammen, ,als das System von Statusfunktionen nicht mehr akzeptiert wurde. Die Furcht vor einer sowjetischen Intervention war nicht länger glaubhaft [...]. “22 Das konstitutive Element, das, Searle zufolge, in diesen beiden Beispielen über die (Nicht-)Persistenz von Grenze und Staat entscheidet, ist die fortdauernde soziale Akzeptanz der zugrundeliegenden Statusfunktionen - und damit die Akzeptanz derjenigen konstitutiven Regeln, die

\footnotetext{
${ }^{20}$ Vgl. auch Searle (2006), S. 17-18.

${ }^{21}$ Searle (2010), S. 94 greift das Beispiel wieder auf.

${ }^{22}$ Searle (1997), S. $102=(1995)$, S. 92.
} 
diese Statusfunktionen begründen. Oder, wie Searle es zusammenfasst:

„Das Geheimnis der fortdauernden Existenz institutioneller Tatsachen besteht einfach darin, daß die direkt beteiligten Individuen der relevanten Gemeinschaft fortfahren müssen, die Existenz derartiger Fakten anzuerkennen und zu akzeptieren." (Searle 1997, S.126 = 1995, S.117)

Bei Searle finden wir aber noch einen zweiten, davon abweichenden Ansatz zur Persistenz von sozialen Sachverhalten. Searle führt nicht nur die Etablierung sozialer Tatsachen, sondern auch ihre Auflösung und damit ihre Persistenz auf das Vorkommen oder Nichtvorkommen von Sprechakten zurück, die sich der Formel „X zählt als Y im Kontext K“ bedienen:

„Die Formel ,X zählt als $Y^{‘}$ gilt sowohl für die Schaffung wie die fortdauernde Existenz des Phänomens, weil die konstitutive Regel ein Mittel zur Schaffung der Tatsachen ist und die Existenz einer Tatsache im allgemeinen dadurch konstituiert wird, daß sie geschaffen und noch nicht zerstört worden ist.“ (Searle 1997, S.128)

Hier ist nicht nur von der Entstehung sozialer Entitäten die Rede (,Schaffung“), sondern auch von ihrer Persistenz (,fortdauernde Existenz“). Die Persistenz wird diesmal aber nicht durch zeitgleich existierende Akzeptanz erklärt, sondern rein vergangenheitsorientiert (,geschaffen und noch nicht zerstört"). Searle greift hier also zu einem ganz anderen Erklärungsmuster für die Persistenz einer sozialen Entität. Sein Beispiel ist in diesem Fall die Ehe:

„So zählt die Durchführung der Zeremonie als Eheschließung, und heiraten und nicht anschließend sterben, sich scheiden lassen oder die Ehe annullieren lassen zählt als verheiratet sein.“" (Searle 1997, S. 128)

Wir können dies bildlich wie folgt schematisieren:

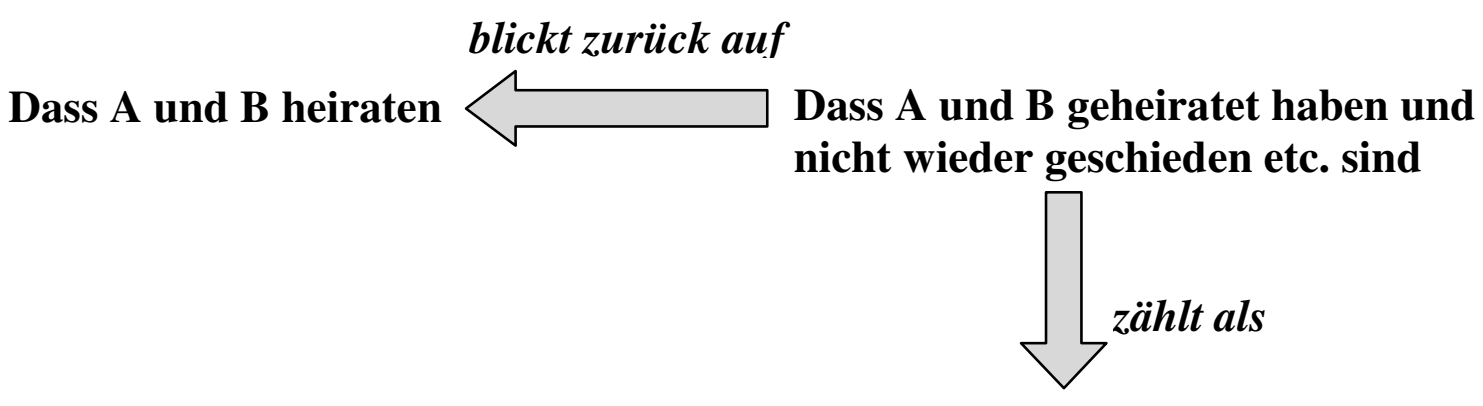

Dass A und B verheiratet sind 
Das Verheiratetsein von A und B wird, diesem Ansatz zufolge, also allein dadurch begründet, dass es ein Entstehungsereignis dieser Relation zwischen A und B gegeben hat, aber kein Auflösungsereignis. Es gibt in diesem Fall keine zeitgleich existierenden Konstituenten des Verheiratetseins. Verheiratetsein ist in diesem Fall eine rein vergangenheitsbezogene Angelegenheit: Es besteht darin, dass A und B in der Vergangenheit an einem Vorkommnis eines bestimmten Ereignistyps beteiligt waren (i. e. der Trauzeremonie), aber nicht an einem Vorkommnis bestimmter anderer Ereignistypen (i.e. einem der möglichen Auflösungsereignisse). ${ }^{23}$

Wir finden bei Searle also zwei unterschiedliche Ansätze für die Analyse der Persistenz sozialer Entitäten. Einmal erklärt er die Persistenz sozialer Kontinuanten durch die Akzeptanz-Abhängigkeit: Eine soziale Entität existiert so lange, wie die direkt beteiligten Individuen der relevanten Gemeinschaft ihre Existenz akzeptieren. Der zweite Erklärungsstrang, der zu diesem hinzutritt, erklärt die Persistenz sozialer Sachverhalte durch ihr Fortdauern bis zur expliziten Auflösung: Dass ein sozialer Sachverhalt fortexistiert heißt nichts anderes, als dass sein Begründungsereignis, aber kein Auflösungsereignis stattgefunden hat. Beide Ansätze sind eingebunden in den weiteren Rahmen von Searles Theorie der Statuszuweisungen durch die Akzeptanz konstitutiver Regeln der Form „X zählt als Y im Kontext $\mathrm{K}^{\prime}$, nur sind es im zweiten Ansatz bestimmte Ereignisse (und keine Kontinuanten), die die entscheidenden Träger des Status „Auflösungsereignis“ sind und deren Vorkommen bzw. Nicht-Vorkommen über die Persistenz der entsprechenden Kontinuanten entscheiden.

\subsection{Gilbert über Spazierengehen und andere Pluralsubjekte}

Wenden wir uns nun Margaret Gilberts Theorie der Pluralsubjekte und dem Problem der Dauer solcher Pluralsubjekte zu. Gilberts Pluralsubjekte sind soziale Kontinuanten, die gewissermaßen durch MiniaturGesellschaftsverträge aus einer Mehrzahl von Einzelpersonen entstehen. Eines von Gilberts Standardbeispielen ist das gemeinsame Spazierengehen - sie bezeichnet es sogar als ,,a paradigmatic social phenomenon“. ${ }^{24}$ Ein

${ }^{23}$ Vgl. Digesten 24, 2, 1: ,Die Ehe wird getrennt durch Scheidung (divortium), Tod, Gefangenschaft, oder wenn eine andere Sklaverei einen von beiden Ehegatten betrifft.“ (Übers. Härtel, 248)

${ }^{24}$ Gilbert (1990), Titel = (1996), S. 177. 
entsprechender „Mini-Gesellschaftsvertrag“ könnte z.B. folgende Form haben:

Anne: „Sollen wir noch einen Spaziergang zum Hafen machen?“

Bernd: „Gute Idee, ich bin dabei!““

Solche Wortwechsel sind uns aus unserem Alltag wohl vertraut. Doch handelt es sich tatsächlich um einen „Vertrag“, der da geschlossen wird? Es ist, so könnten wir erwidern, zumindest etwas Vertragsähnliches - denn durch solche alltäglichen Wortwechsel erwachsen den Beteiligten Sanktionsmöglichkeiten: ${ }^{25}$ Sollte Anne nicht die Richtung zum Hafen einschlagen, sondern stattdessen zum Bahnhof spazieren, könnte Bernd mit gutem Recht protestieren. Anne und Bernd sind, so Gilbert, eine gemeinsame Verpflichtung (ein joint commitment) eingegangen, zum Hafen zu spazieren. Sie haben gegeneinander ein Recht auf und eine Verpflichtung zu einem Spaziergang zu diesem Ziel mit dem jeweils anderen. Damit wird aus dem Personenkollektiv Anne und Bernd ein durch deontische Strukturen zusammengehaltenes Pluralsubjekt (plural subject) für eine ganz bestimmte Handlung, nämlich den gemeinsamen Spaziergang.

Ähnlich wie bei Searles Vorschlag zur Analyse der Eheschließung steht auch am Anfang eines Gilbert'schen Pluralsubjekts ein Sprechakt (oder eine Reihe von Sprechakten), durch den die Beteiligten wechselseitig ihre Bereitschaft zur Beteiligung signalisieren und die Beteiligung der anderen akzeptieren. Gilbert weist nun aber darauf hin, dass die Existenz des Pluralsubjekts keine rein vergangenheitsbezogene Angelegenheit ist: Das ÄuBern der Sprechakte bringt nicht nur das Pluralsubjekt selbst hervor, sondern auch die Verpflichtungen der Beteiligten, zum gemeinsamen Projekt beizutragen. Und diese Verpflichtungen dauern an, auch wenn die sie hervorbringenden Sprechakte bereits in der Vergangenheit versunken sind. Und entsprechend existiert ein Pluralsubjekt genau so lange, wie die beteiligten Personen durch die das Pluralsubjekt konstituierenden deontischen Beziehungen miteinander verbunden sind:

${ }^{25}$ Vgl. Gilbert (1990), S. 3-5 = (1996) S. 180-182. Gilbert diskutiert verschiedene Möglichkeiten, ein Pluralsubjekt zu etablieren. Eine explizite Vereinbarung ist für sie dabei nicht notwendig (vgl. Gilbert, 1989, S. 381), wohl aber hinreichend, und das ist für den gegenwärtigen Kontext ausreichend. 
Der Mini-Gesellschaftsvertrag von A, B und C

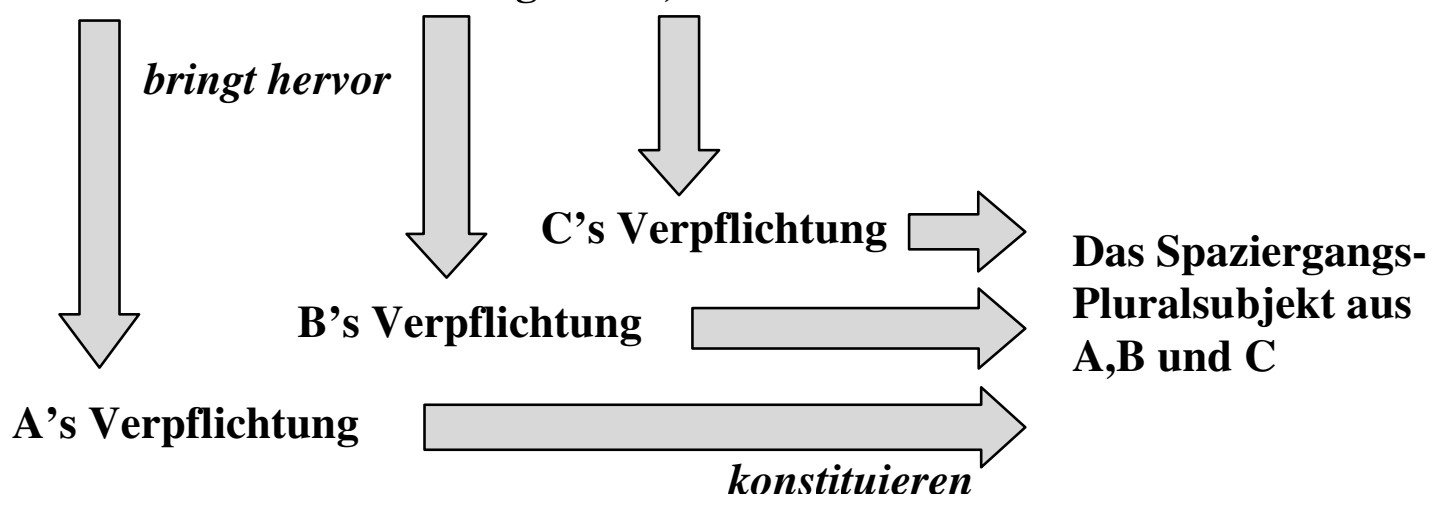

Pluralsubjekte können neue Mitglieder aufnehmen: Durch „Erweiterungsverträge“" kann das ursprüngliche joint commitment auf neue Beteiligte ausgedehnt werden. Und Pluralsubjekte können durch „Änderungsverträge" das joint commitment variieren oder ergänzen: Wenn Anne und Bernd während des Spaziergangs zum Hafen beschließen, während eines Stücks des Weges gemeinsam zu singen, dann hat sich ein und dasselbe Pluralsubjekt zum Subjekt einer weiteren Handlung entwickelt. Aber nicht immer sind mehrere Verpflichtungen, die dieselben Beteiligten untereinander verbinden, demselben Pluralsubjekt zuzuschreiben: Dieselben Beteiligten könnten sowohl eine Sportgruppe bilden, die sich montags trifft, wie auch einen Literaturzirkel, der sich mittwochs trifft, und so zwei unabhängig voneinander existierende Pluralsubjekte bilden. Hier haben wir es nicht mit einer Änderung oder Ergänzung des ursprünglichen Miniatur-Gesellschaftsvertrags zu tun, sondern mit einer ,Zweitgründung“. ${ }^{26}$

Viele Pluralsubjekte sind, wie das Spaziergangs-Pluralsubjekt aus Anne und Bernd, Subjekte für partikuläre Handlungen (oder Komplexe aus partikulären Handlungen), in unserem Beispiel für einen bestimmten Spaziergang zum Hafen. Sie existieren genau so lange, wie die Verpflichtungen zu diesen Handlungen existieren. Mit der Vollendung der entsprechenden Handlungen hört die Verpflichtung dazu auf zu existieren, und mit dem Erlöschen der Verpflichtung hört das Pluralsubjekt auf zu sein. Anders als Staaten, die über Jahrhunderte hinweg existieren können, haben wir es bei einem Spaziergangs-Pluralsubjekt also mit einem sehr vergänglichen Phä-

${ }^{26} \mathrm{Zu}$ Erweiterungen, Änderungen und Zweitgründungen vgl. Gilbert (1989), S. 219221. 
nomen zu tun. Gilbert'sche Pluralsubjekte können also äußerst ephemere Entitäten sein, ${ }^{27}$ die mitunter nur eine einzige Handlung ausüben, bevor sie aufhören zu existieren.

Ein Pluralsubjekt hört auf zu existieren, wenn die das Pluralsubjekt konstituierenden Verpflichtungen zu existieren aufhören. Dies kann erstens durch Erfüllung ebendieser Verpflichtungen geschehen: Wenn Anne und Bernd gemeinsam zum Hafen spaziert sind, haben sie ihr joint commitment erfüllt. Zweitens kann das Ende einer Verpflichtung explizit durch einen Auflösungsvertrag herbeigeführt werden. Wenn Anne und Bernd nach einiger Zeit vereinbaren, nun doch nicht zum Hafen zu gehen, sondern den gemeinsamen Spaziergang vorher abzubrechen, hört ihr joint commitment auf zu existieren und sie sind ihrer zuvor eingegangenen Verpflichtungen ledig. $^{28}$

Diese beiden Weisen, die Existenz eines Pluralsubjekts zu beenden, können als zwei Weisen der „Beendigung“ des Miniatur-Sozialvertrags verstanden werden. ${ }^{29}$ Die Beendigung des Vertrags kann in seiner Erfüllung oder seiner Auflösung bestehen. Weitere Formen der Beendigung eines Vertrags sind die Überleitung in einen anderen Vertrag oder die Befristung der wechselseitigen Verpflichtung schon bei der Etablierung des Pluralsubjekts. Wie steht es nun, wenn einen (oder mehrere) der Beteiligten der Tod ereilt und er als Verpflichtungsinhaber nicht mehr zur Verfügung steht? Im Falle des Spaziergangs sollte man meinen, dass die Verpflichtung damit ebenfalls aufhört zu existieren. Doch dies ist nicht bei allen Verpflichtungen der Fall: Die Verpflichtung, jemandem ein Darlehen zurückzuzahlen, geht vom Darlehensnehmer auf dessen Erben (oder Rechtsnachfolger) über, und entsprechend wird das Recht auf Rückzahlung vom Darlehensgeber an seine Erben (oder Rechtsnachfolger) weitergegeben.

\subsection{Noch einmal Searle}

Gilberts These, der gemeinsame Spaziergang zweier Freunde habe ein ihm

27 Vgl. Gilbert (1989), S. 215 (,ephemeral and small“, „ephemeral conversational groups“). Schon Weber (1913), S. 448 unterscheidet „ephemere“ und ,perennierende“ soziale Gebilde.

${ }^{28}$ Vgl. Gilbert (2000), S. 26-27.

${ }^{29}$ Diesen Punkt verdanke ich einem Hinweis von Pedro Schmechtig. 
eigentümliches Subjekt, nämlich das aus Anne und Bernd bestehende Pluralsubjekt dieses Spaziergangs, mag vielen überzogen vorkommen. Vielleicht hat Searle Gilberts Ausführungen zum Spazierengehen vor Augen, wenn er an einer Stelle seines Buches schreibt:

„In meiner Gesellschaft nennt man , mit jemandem spazierengehen' eine gesellschaftliche, aber keine institutionelle Tatsache, weil dieses Etikett keine neuen Statusfunktionen zuweist. Es etikettiert einfach die Intentionalität und ihre Manifestation." (Searle 1997, S.98 $=1995$, S.88) ${ }^{30}$

Die Menge der gesellschaftlichen Tatschen (der social facts) ist für Searle wesentlich größer als die Menge der institutionellen Tatsachen (der institutional facts). ${ }^{31}$ Erstere kommen schon im Tierreich vor, wenn Tiere sich etwa gemeinsam um die Brutpflege kümmern oder wenn Wölfe in Rudeln jagen. Institutionelle Tatsachen hingegen entstehen erst durch die Akzeptanz von Statuszuweisungen durch konstitutive Regeln; sie setzen Sprache voraus und kommen nach allem, was wir wissen, erst in der menschlichen Sphäre vor. Gemeinsames Spazierengehen ist für Searle also ein social fact, aber kein institutional fact, eher vergleichbar dem Jagen des Wolfsrudels als solchen Parade-Institutionen wie Staat und Ehe. Doch wenn wir Gilberts Beobachtung der Sanktionierbarkeit devianten Verhaltens in Pluralsubjekten ernst nehmen, dann müssen wir Searles Diagnose widersprechen: Denn dann geht bereits ein gemeinsamer Spaziergang mit Rechten und Pflichten einher, nämlich mit dem Recht auf die Begleitung des anderen und der Pflicht, den Anderen zu begleiten.

Schon die Verabredung eines gemeinsamen Spaziergangs geht also mit der Schaffung deontischer Strukturen einher, und damit, wie Searle sagt, mit der Schaffung von Statusfunktionen:

„Weil die Schaffung einer Statusfunktion auf der Zuweisung eines Status und damit einer Funktion an ein Gebilde beruht, das diese Statusfunktion nicht schon hat, hängt die Schaffung einer Statusfunktion im allgemeinen von der Übertragung einer neuen Macht (power) ab.“ (Searle 1997, S. $105=1995$, S. 95)

Vor diesem Hintergrund können wir nun aber zwanglos Gilberts Modell

30 Searle ist recht sparsam mit Literaturangaben; er erwähnt Gilbert (1989) aber in Anmerkung 7 zu Kapitel 1; vgl. Searle (1997), S. $239=$ (1995), S. 230.

${ }^{31}$ Vgl. etwa das Taxonomie-Schema bei Searle (1997), S. $131=(1995)$, S. 121. 
der Persistenz von Pluralsubjekten auch auf Searles Ehe-Beispiel übertragen:

\section{Das Eheversprechen von A und B}

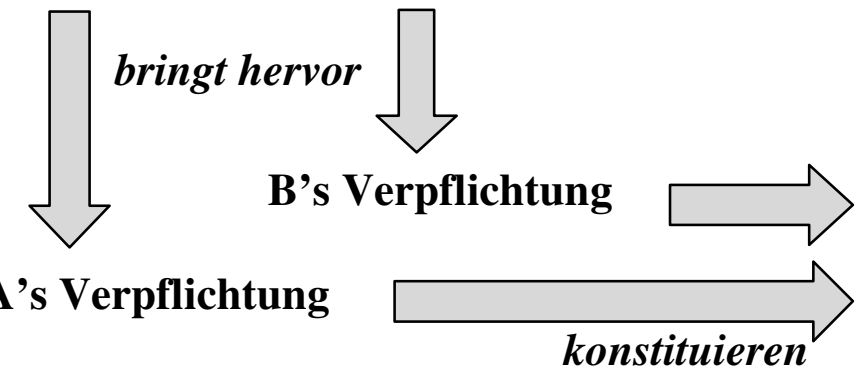

A's und B's Verheiratetsein

Wir können die Persistenz einer Ehe nun ganz analog zum SpaziergangsBeispiel erklären: Das bei der Eheschließung gegebene wechselseitige Versprechen der Heiratenden bringt die wechselseitigen Verpflichtungen der Eheleute hervor, und diese wechselseitigen Verpflichtungen dauern fort, wenn die Äußerung der entsprechenden Sprechakte vorüber ist. ${ }^{32}$ Es sind diese Verpflichtungen, die die Ehe konstituieren, und die Eheleute sind genau so lange miteinander verheiratet, wie sie durch diese Verpflichtungen miteinander verbunden sind. ${ }^{33}$ In dieser Perspektive ist das Verheiratetsein nicht länger eine bloße vergangenheitsbezogene Angelegenheit: Nicht allein, weil bestimmte Ereignisse zurückliegen und andere nicht geschehen sind, sind zwei Menschen miteinander verheiratet, sondern weil durch ein solches Ereignis bestimmte deontische Strukturen entstanden sind, die die gesamte Zeit ihres Verheiratetseins fortdauern und dieses konstituieren. $^{34}$

\footnotetext{
${ }^{32}$ Vgl. Reinach (1913), § 4, S. $743=(1989)$ S. 188, der sagt, ,,daß das Versprechen als solches Anspruch und Verbindlichkeit erzeugt", und Searle (1969), S. 179: „All promises are acts of placing oneself under (undertaking) an obligation to do the thing promised."

${ }^{33}$ Das bei der Eheschließung gegebene Versprechen ist nicht zu verwechseln mit dem einem Verlöbnis entsprechenden Versprechen einer zukünftigen Eheschließung. Diese Unterscheidung ist schon recht alt; vgl. z.B. Baxter (2007): „Roman law during the period of the empire distinguished between an agreement for present marriage and an agreement for future marriage (sponsalia per verba de praesenti and sponsalia per verba de futuro)."

${ }^{34}$ Noch deutlicher sind die Affinitäten zu Gilbert in Searle (2010), wo Searle immer wieder die konstitutive Rolle von „obligations“ (S. 48) oder ,power relations“ (z.B. S. 106) betont.
} 


\section{Das Ende sozialer Kontinuanten}

Wir haben nun schon einige Möglichkeiten kennengelernt, wie soziale Kontinuanten im Allgemeinen und Pluralsubjekte im Besonderen aufhören können zu existieren. Erstens können soziale Entitäten durch Auflösungsakte aufhören zu existieren: Ein Scheidungsurteil beendet die Existenz von Ehen, und ein Vereinigungsvertrag kann die Existenz eines Staates beenden. Auch eine politische Grenze kann durch einen Vertrag (etwa einen Vereinigungsvertrag) aufhören zu existieren. Pluralsubjekte, die nach Gilberts Analyse ja durch wechselseitige Verpflichtungen konstituiert werden, können darüber hinaus auch schlicht dadurch aufhören zu existieren, dass die ihnen zugrundeliegende wechselseitige Verpflichtung erfüllt wird. Das Pluralsubjekt eines Spaziergangs hört nach dem Ende des Spaziergangs auf zu existieren. Eine Ehe wird geschlossen, wie es so schön heißt, „bis das der Tod sie scheidet"; stirbt aber einer der Ehepartner, dann ist damit die wechselseitige Verpflichtung erfüllt, die die Ehepartner bei der EheschlieBung eingegangen sind, und die Ehe hört auf zu existieren. ${ }^{35}$

Eine weitere Möglichkeit für das Ende einer sozialen Entität, die wir bisher noch nicht erörtert haben, ist die Auflösung durch eine höhere Instanz: Eine Partei kann beispielsweise dadurch aufhören zu existieren, dass sie durch den Staat, in dem sie wirkt, verboten wird. Analog könnte ein Staat, der sich nicht liberalen Prinzipien verpflichtet hat, auch Ehen für nichtig erklären und so auf dem Wege des Verbots das Ende der Existenz solcher sozialen Entitäten herbeiführen. Und ein Stück Papier hört auf, ein Geldschein zu sein, wenn die ausgebende Instanz diesen z.B. durch einen entsprechenden Stempelaufdruck entwertet.

Neben diesen drei Wegen der Erfüllung, des Auflösungsaktes und des Verbotes werden oft auch andere Wege genannt, die Existenz einer sozialen Entität zu einem Ende zu bringen. Drei weitere Kandidaten werde ich nun diskutieren: Vergessen, Gewalt und Protest. Ich werde zeigen, dass diese Wege zwar wichtige kausale Einflüsse darstellen, dass sie aber nicht selbst Auflösungsereignisse für soziale Entitäten sind.

\footnotetext{
35 Die Institution der Ehescheidung ändert an dem hier wichtigen Punkt nichts: Der Tod eines Gatten ist hinreichend für das Ende einer Ehe, er ist dafür allerdings nicht notwendig.
} 


\subsection{Ende durch Vergessen?}

„In gewissem Sinne“, schreibt Searle in seinem Buch Die Konstruktion der gesellschaftlichen Wirklichkeit, ,gibt es Dinge, die nur existieren, weil wir glauben, daß sie existieren." Searle denkt dabei, so schreibt er, ,an Dinge wie Geld, Eigentum, Regierungen und Ehen“ ${ }^{36}$ Diese Formulierung suggeriert, dass es lediglich von den richtigen Überzeugungen, also von kognitiven Akten, ${ }^{37}$ abhängt, ob man reich und mit seinem Traumpartner verheiratet ist und man von seiner bevorzugten Partei regiert wird (oder man gar selbst Regierungsmitglied ist). ${ }^{38}$ Und tatsächlich sieht Searle das Ende sozialer Entitäten als gekommen an, wenn niemand mehr ihre soziale Funktion kennt:

„Wenn jeder aufhört zu glauben, daß es sich [bei dem Gegenstand in meiner Tasche] um Geld handelt, hört es auf, als Geld zu fungieren, und hört schließlich auf, Geld zu sein." (Searle 1997, S. $42=1995$, S. 32$)^{39}$

Das, was Searle am Beginn seines Buches als Paradox beschreibt, ist aber schlicht keine gute Deskription der gesellschaftlichen Wirklichkeit: Nicht kognitive Akte machen mich reich, sondern hohe Einzahlungen auf mein Bankkonto, und nicht die richtigen Überzeugungen machen mich zum Ehemann meiner Traumfrau, sondern ein beiderseitiges „Ja“ vor dem Standesbeamten. Und auch eine Regierung wird nicht durch kognitive Akte konstituiert, sondern durch komplexe Wahl- und Ernennungsverfahren, die nicht nur von Land zu Land variieren, sondern zudem noch einem historischen Wandel unterworfen sind.

Das „Ja“ vor dem Standesbeamten und das Kreuz auf dem Wahlzettel sind keine kognitiven, sondern volitionale Akte. Es sind Absichtserklärun-

\footnotetext{
${ }^{36}$ Searle (1997), S. $11=(1995)$, S. 1.

37 Mindestens ein Rezensent hat Searle so gelesen; vgl. Zaibert (1998), S. 203 („,facts which exist in virtue of human cognition“").

${ }^{38} \mathrm{Vgl}$. auch Searle (2006), S. 14: ,Suppose I now think, ,This is a \$20 bill'. That attitude and countless others like it are constitutive of the observer-relative fact that things of this sort are money."

${ }^{39}$ Wenige Sätze später ergänzt Searle: „Wenn jeder immer glaubt, daß diese Art von Ding Geld ist, und alle es als Geld benutzen und es als Geld behandeln, dann ist es Geld. Wenn keiner jemals glaubt, daß diese Art von Ding Geld ist, dann ist es kein Geld." Doch hier mogelt er sich um die interessanten Fälle herum: Was ist bei Dissens, und was ist bei dem zuvor von ihm skizzierten Vergessen?
} 
gen oder Willensäußerungen: Ich will mit dieser Frau verheiratet sein, ich will von dieser Partei regiert werden. Wenn meine Auserwählte dem Standesbeamten eine korrespondierende Willensäußerung kundgibt, ist die Ehe erfolgreich geschlossen. Meine Wunschpartei hat ihren Willen, mich zu regieren, bereits durch den Antritt zur Wahl kundgetan; in diesem Fall hängt die erfolgreiche Wahl aber von den Willensäußerungen der Wähler ab. In beiden Fällen sind es aber nicht kognitive, sondern volitionale Akte, die Ehen und Regierungen hervorbringen. ${ }^{40}$ Solche volitionalen Akte können einseitige oder wechselseitige Versprechen sein (die letzteren nennt man auch Verträge), es können Abstimmungen oder Wahlen sein, sie können mündlich oder schriftlich verfasst sein, formlos oder ritualisiert erfolgen, ohne Publikum oder vor Zeugen oder vor staatlichen Autoritäten ausgeführt werden.

Wenn nun beispielsweise für eine Ehe kognitive Akte nicht konstitutiv sind, dann sollten kognitive Defekte die Existenz einer Ehe auch nicht gefährden. Sollte beispielsweise ein Ehemann vergessen, dass er verheiratet ist, würden wir kaum die Ehe als nicht-existent ansehen. ${ }^{41}$ Ein solches einseitiges Vergessen und das daraus resultierende Verhalten würden vermutlich, sofern es durch Nachlässigkeit und nicht durch eine Krankheit oder einen Unfall verursacht ist, moralisch getadelt und entsprechend sanktioniert werden. Was für eine solche einseitige Amnesie durch den Ehemann gilt, gilt ganz analog auch für die einseitige Amnesie der Eheschließung durch die Ehefrau. Und nicht nur kann jeder der Eheleute je für sich die Eheschließung vergessen, ohne dass die Ehe aufhört zu existieren, sondern auch beide gemeinsam können ihre Eheschließung vergessen, ohne dass die Ehe aufhört zu existieren. ${ }^{42}$ Die Ehe könnte auch eine solche beidseitige Amnesie überleben. Beispielsweise kann es immer noch Standesbeamte

${ }^{40}$ Durch volitionale Akte hervorgebrachte Dinge wurden auch als entia moralia bezeichnet; zur Geschichte dieses Stranges der abendländischen Metaphysik vgl. Kobusch (1993).

41 Ähnliches sieht das römische Recht für den Fall der „Geisteskrankheit“"vor; vgl. Digesten I, 6, 8: „Der Geisteskranke kann zwar keine Frau heiraten, aber eine Ehe fortsetzen“ (Übers. Härtel, S. 234).

${ }^{42}$ Analog wieder das römische Recht für den Fall der „Geisteskrankheit“; vgl. Digesten I, 6, 8: „Denn wenn die Ehe fortbesteht, wenn einer der Ehegatten geisteskrank ist, so muß sie auch fortbestehen, wenn beide geisteskrank sind" (Übers. Härtel, S. 235). 
und Hochzeitsgäste geben, die sich an die Eheschließung erinnern, und obendrein Familienbücher und Heiratsregister, die sie dokumentieren. Doch was passiert bei einer Hyper-Amnesie, bei der nicht nur alle Gedächtnisspuren, sondern auch alle Registratureinträge und Urkunden zerstört werden, die von der Eheschließung Zeugnis geben könnten?

Praktisch gesehen würde niemand mehr davon ausgehen, dass unsere beiden Eheleute miteinander verheiratet sind. Sie würden nicht das Leben eines Ehepaares führen und auch nicht mehr die mit diesem Status verbundenen Rechte in Anspruch nehmen können, da ihre Ehe nirgendwo dokumentiert ist. Aber würden Sie nicht mehr verheiratet sein? Die wechselseitigen Verpflichtungen, die sie bei der Eheschließung eingegangen sind, sind weder aufgelöst noch erfüllt. Sie bestehen weiter fort, und mit ihnen die Ehe: Praktisch hat sich alles geändert, ontologisch hingegen hat sich nichts geändert. Ganz analog können wir bei Statusentitäten wie Geldscheinen oder politischen Grenzen argumentieren: Eine Hyper-Amnesie, die alle Erinnerungen und Dokumentationen über ihren Status verschwinden lässt, würde ihrem Status zwar jede praktische Relevanz nehmen. Aber die deontischen Strukturen, die sie konstituieren, fallen damit noch nicht weg. Ansonsten wären wir gezwungen, bei einem Vergehen der HyperAmnesie nach wenigen Minuten ganz viele Entstehungsereignisse anzunehmen: Alle Ehen, Staaten, Geldscheine, politischen Grenzen und was auch immer von der Hyper-Amnesie betroffen war, würden dann wieder entstehen.

\subsection{Ende durch Gewalt?}

Wie sieht es nun aus mit der Androhung und eventuell sogar Anwendung von Gewalt? Können soziale Entitäten durch Gewalt zerstört werden? Das Zerstörungspotential moderner Waffen ist gewaltig; der nukleare overkill erlaubt es, menschliches Leben auf unserem Planeten mehrfach auszulöschen, und damit das gesamte soziale Leben und alle sozialen Institutionen. Auf diese Weise könnte man natürlich auch das Ende einer Ehe herbeiführen, indem man das Ende der Eheleute herbeiführt. Schon der Tod einer der Eheleute bringt ja das Ende der Ehe mit sich. Analog können Geldscheine dadurch ihr Ende finden, dass man ihr materielles Substrat verbrennt.

Geht es auch sparsamer? Kann die Ehe durch Gewalteinwirkung aufhö- 
ren zu existieren, ohne dass die Eheleute zu existieren aufhören? Die Ehe wird konstituiert durch die wechselseitigen Verpflichtungen, die Mann und Frau miteinander verbinden, und keine Waffe der Welt kann Verpflichtungen zerstören, ohne dass sie die daran beteiligten Personen tötet. Aber können die Verpflichtungen vielleicht durch die Androhung von Gewalt gegen die Eheleute oder Dritte zerstört werden? Nehmen wir an, unsere Eheleute würden erpresst: Wenn Sie sich nicht scheiden ließen, würden die Erpresser mitten in einer Großstadt eine Atombombe detonieren lassen. Wir können annehmen, dass unsere Eheleute bereit wären, diese Berohung dadurch abzuwenden, dass sie die Scheidung beantragen. Das Ende der Ehe tritt dann aber nicht durch die Gewaltandrohung ein, sondern durch das Scheidungsurteil. Die Androhung von Gewalt kann also nicht selbst das Ende von sozialen Entitäten herbeiführen. Sie kann aber dazu führen, dass entsprechende Auflösungsakte eingeleitet werden.

Ganz ähnlich verhält es sich, wenn ein Staat versucht, durch einen Krieg eine politische Veränderung herbeizuführen. Der Krieg ist beendet, wenn einer der Gegner seine Kapitulation erklärt. Der Krieg kann auch dann nicht fortgeführt werden, wenn es auf der Seite des Kriegsverlierers gar keine Überlebenden gibt - und damit niemanden mehr, gegen den man kämpfen könnte, auch wenn es dadurch niemanden gibt, der die Kapitulation aussprechen kann. Vielleicht ist ein solches Szenario die einzige Möglichkeit, den sogenannten war on terror zu gewinnen, der nicht gegen einen Staat, sondern gegen ein soziales Phänomen geführt wird. Denn anders als Staaten haben soziale Phänomene gemeinhin keine Sprecher, die eine Kapitulationserklärung aussprechen könnten. Aber sehen wir hier von diesen Sonderfällen ab. Nach der Kapitulation des Verlierers kann der Gewinner seine politischen Vorstellungen entweder per Dekret oder durch einen Friedensvertrag politisch durchsetzen. Auch hier gilt: Kriegsführung und Niederlage des Gegners sind Ursachen dafür, dass der Gewinner in der Lage ist, ein solches Dekret zu erlassen bzw. der Verlierer bereit ist, einen solchen Friedensvertrag abzuschließen. Aber die Verwirklichung der politischen Veränderung erfolgt erst durch das Dekret oder den Vertragsabschluss. Und auch eine Geldentwertung oder das Verschwinden einer Grenze kann nicht durch Gewalt oder Gewaltandrohung geschehen, sondern nur durch einen - möglicherweise durch Gewalt oder Gewaltandrohung herbeigeführten - Auflösungsakt. 


\subsection{Ende durch Protest?}

Der Protest als dritter Kandidat kann nun relativ schnell abgehandelt werden. Ebenso wie Gewalt und die Drohung mit Gewalt spielt der Protest im politischen Leben eine wichtige Rolle: Politischer Protest intendiert, staatliche Ordnungsstrukturen zu beeinflussen oder zu ändern, und manchmal, wie im annus mirabilis 1989, haben diese Proteste einen erstaunlichen Erfolg. Aber wiederum gilt: Die Veränderungen treten nicht durch den Protest selbst ein, sondern werden von ihm vorbereitet und verursacht.

Analog könnten wir uns im Falle unseres Ehepaares vorstellen, dass es (um ein altes Klischee zu bedienen) zu massiven Protest-Interventionen von Seiten der Schwiegermutter kommt. Wiederum können die Proteste der Schwiegermutter aber nicht selbst das Ende der Ehe herbeiführen, sondern, nicht anders als die Gewaltandrohung, lediglich einen Auflösungsakt nur motivieren. Gleiches gilt für die Geldentwertung und das Verschwinden der politischen Grenze. Auch Geldscheine und Grenzen hören nicht durch Proteste auf zu existieren, sondern durch von Protesten ausgelöste Auflösungsakte.

\subsection{Psycho-soziale vs. ontologische Analysen}

Wir haben gesehen, dass weder Vergessen noch Gewaltandrohung oder Protest für sich genommen in der Lage sind, die Existenz sozialer Entitäten zu beenden. Freilich: Vergessen, Gewalt und Protest können einem Ehepaar das Leben schwer machen. Sie können zum Ehestreit führen, dazu, dass die Eheleute sich ,,auseinanderleben“, dass sie sich entfremden etc.

All dies ist nun einerseits mit einem Fortbestehen der Ehe logisch und metaphysisch kompatibel. All dies macht es aber auch wahrscheinlicher, dass mindestens einer der Eheleute ein Interesse daran bekommt, den Rechtszustand des Verheiratetseins aufzuheben, und deshalb die Scheidung beantragt. Wir haben es deshalb mit sozialen und psychischen Ursachen des Endes von Ehen zu tun - während die ontologische Analyse ihr Ende allein dort verortet, wo die die Eheleute verbindende deontische Struktur, also ihre wechselseitigen Verpflichtungen, aufhören zu existieren.

Dieses Ergebnis können wir auf die politische Sphäre übertragen. Auch dort sind Vergessen, Protest und Gewalt sozial und psychisch relevant, nicht aber ontologisch: Denn allein das Abnehmen der sozialen Akzeptanz, das in den wöchentlichen Montagsdemonstrationen zum Ausdruck kam, 
hat noch nicht ausgereicht, um der staatskommunistischen DDR ein Ende zu bereiten. Sie bereiteten aber dadurch den Boden für ihr Ende, dass sie die Politiker der betroffenen Staaten (insbesondere natürlich die Politiker der DDR selbst) dazu motivierten, die Zwei-plus-vier-Gespräche aufzunehmen, in denen Vertreter der beiden deutschen Staaten und der vier im Zweiten Weltkrieg siegreichen Alliierten über die Zukunft der deutschen Staaten berieten. Und erst durch die dort beschlossenen und unterzeichneten staatsrechtlichen Vereinbarungen konnte die DDR am 3. Oktober 1990 aufhören zu existieren. Ohne den vorhergehenden Protest wäre dies nicht möglich gewesen: Das ist die sozio-politische Analyse des Historikers. In ontologischer Perspektive ist es aber der Auflösungsakt durch den Einigungsvertrag, der die politischen Strukturen tatsächlich aufgelöst hat.

Neben die ontologische Analyse des Endes sozialer Entitäten tritt also die Analyse der sozialen, politischen oder psychischen Ursachen für ihr Ende. Dazu gehört die Analyse derjenigen Faktoren, die für das Initiieren und das Aufrechterhalten oder aber das Hemmen und Verändern individueller und kollektiver Einstellungen verantwortlich sind - oder, wie man auch sagt, zur Stiftung und Stärkung einer entsprechenden kollektiven Identität. Dazu gehören das Symbolisieren, Memorieren, Habitualisieren, Tradieren, Verschriftlichen und Archivieren der entsprechenden Grundlagen der betroffenen Institutionen, ihrer Rechte und Strukturen. ${ }^{43}$

\section{Ergebnisse}

In diesem Aufsatz habe ich die Dauer sozialer Kontinuanten vor dem Hintergrund verschiedener Konstitutionsmodelle sozialer Entitäten untersucht. Die ontologischen Konstituenten sind als „Prinzipien des Seins“ unterschieden sowohl von den Ursachen der Etablierung sozialer Entitäten, also ihren „Prinzipien des Werdens“, als auch von Ursachen anderer Bereiche, etwa psychologischen, sozialen oder politischen Ursachen. Die Arbeitshypothese, von der ich ausgegangen bin, war, dass soziale Kontinuanten so lange (und deshalb) fortdauern, solange (und weil) diejenigen ihrer Konstituenten fortdauern, von denen sie rigide ontologisch abhängig sind, bzw.

\footnotetext{
43 Nicht ganz zufällig gibt es in dieser Aufzählung große Überschneidungen mit den Arbeitsgebieten des Dresdener Sonderforschungsbereiches „Institutionalität und Geschichtlichkeit““. Für erste Hinweise vgl. z.B. Vorländer und Melville (2002).
} 
solange (und weil) Konstituenten ebender Art existieren, von denen sie generisch abhängig sind.

Zunächst habe ich zwei Standardtheorien der Sozialontologie zu ihrer Auskunft über die Persistenz von sozialen Kontinuanten befragt. Bei John Searle konnten wir gleich zwei unterschiedliche Modelle für die Persistenz sozialer Kontinuanten entdecken: ein akzeptanztheoretisches Modell und ein vergangenheitsbezogenes Modell. In Margaret Gilberts Theorie der Pluralsubjekte habe ich deontische Strukturen als die entscheidenden Konstituenten eines Pluralsubjekts herausgearbeitet, und ich konnte zeigen, dass dieses Modell auch auf die Fälle anwendbar ist, mit denen Searle sein zweites Modell stützt. Wir können damit Searles bloß vergangenheitsbezogenes Modell durch ein echtes Konstitutionsmodell à la Gilbert (mit zeitgleich existierenden Konstituenten) ersetzen. Zweitens habe ich gezeigt, dass Faktoren wie Vergessen, Protest und Gewalt und ihre positiven Gegenstücke Erinnerung, Akzeptanz und Freiheit nur die sozialen Ursachen des Endes oder der Fortdauer sozialer Entitäten bilden, nicht aber ihre ontologischen Konstituenten. Dann aber ist der erste von Searles beiden Vorschlägen keine ontologische Erklärung des Endes einer sozialen Entität, sondern eine soziologische Kausalerklärung. Die Dopplung der Modelle bei Searle könnte dann damit erklärt werden, dass er die sozialen und die ontologischen Ursachen nicht klar genug auseinander hält.

\section{Literatur}

Aristoteles: Politica. Hg. v. W. D. Ross, Oxford, 1957.

Baker, Lynne Rudder: The Metaphysics of Everyday Life. An Essay in Practical Realism. Cambridge, 2007.

Baxter, Ian F. G.: Family law. In: Encyclopadia Britannica. Encyclopadia Britannica 2007 Deluxe Edition, CD-ROM, Chicago, 2007.

Doepke, Frederick C.: The Kinds of Things. A Theory of Personal Idenitity

Based on Transcendental Argument. Chicago/La Salle IL, 1996.

Ferraris, Maurizio: Dove Sei? Ontologia del Telefonino. Mailand, 2005.

Gilbert, Margaret: On Social Facts, zuerst London, New York, 1989; ND: Princeton, NJ, 1992.

Gilbert, Margaret: Walking Together: A Paradigmatic Social Phenomenon. In: P. A. French et al. (Hg.): The Philosophy of the Human Sciences (= 
Midwest Studies in Philosophy 15), Notre Dame, 1990, 1-14; ND in ders.: Living Together, 1996, 177-194.

Gilbert, Margaret: What Is It for $U s$ to Intend? In: dies.: Sociality and Responsibility. New Essays in Plural Subject Theory 6. Lanham, 2000, 14-36.

Härtel, Gottfried (Übers.): Aus den Digesten. In: L. Huchthausen, G. Härtel (Hg.): Römisches Recht. 225-306, Berlin, Weimar, 1975.

Jansen, Ludger: Institutionen und die kategoriale Ontologie. In: G. Schönrich (Hg.): Instutionen und ihre Ontologie. 45-57, Frankfurt, Lancaster, 2005.

Jansen, Ludger: The Diachronic Identity of Social Entities. In: C. Kanzian (Hg.): Persistence. Frankfurt, 2007, 49-71.

Jansen, Ludger: Kategorien: Die top level Ontologie. In: L. Jansen, B. Smith (Hg.): Biomedizinische Ontologie. Wissen strukturieren für den Informatik-Einsatz.Zürich, 2008, 85-112.

Jansen, Ludger: Vernünftiger Rede fähig. Aristoteles über den Menschen. In:

L. Jansen, C. Jedan (Hg.): Philosophische Anthropologie in der Antike.Frankfurt, Lancaster, 2010, 157-184.

Johansson, Ingvar: Qualities, Quantities and the Endurant-Perdurant Distinction in Top-Level Ontologies. In: G. Büchel, B. Klein, T. RothBerghofer (Hg.), Proceedings of the Second International Workshop on Philosophy and Informatics (= CEUR Workshop-Proceedings 130), online unter: http://CEUR-WS.org/Vol-130/, 2005.

Johnson, William E.: Logic. Part I. Cambridge, 1921.

Kobusch, Theo: Die Entdeckung der Person. Metaphysik der Freiheit und modernes Menschenbild. Freiburg, Basel, Wien, 1993.

Lewis, David: Philosophical Papers, volume II. Oxford, 1986.

Locke, John: An Essay concerning Human Understanding. Hg. von P. H. Nidditch, Oxford, 1975.

Lowe, E. Jonathan: Ontological Dependence. In: E. N. Zalta (Hg.): The Stanford Encyclopedia of Philosophy. Fall 2008 Edition, http://plato.stanford.edu/archives/fall2008/entries/dependenceontological/, 2008.

Reinach, Adolf: Die apriorischen Grundlagen des bürgerlichen Rechtes. In: E. Husserl et al. (Hg.): Jahrbuch für Philosophie und phänomenologische Forschung. 1/II, Halle, 1913, 685-847. ND In: A. Reinach: 
Sämtliche Werke. Textkritische Ausgabe, Bd 1: Werke. Hg. v. K.

Schuhmann, B. Smith, München, 1989, 141-278.

Searle, John R.: Speech Acts: An Essay in the philosophy of language. London, 1969.

Searle, John R.: The Construction of Social Reality. New York, 1995.

Searle, John R.: Die Konstruktion der gesellschaftlichen Wirklichkeit. Zur Ontologie sozialer Tatsachen. Übers. v. M. Suhr, Reinbeck b. Hamburg, 1997.

Searle, John R.: Social ontology. Some basic principles. In: Anthropological Theory 6, 2006, 12-29.

Searle, John R.: Making the Social World. The Structure of Human Civilization. Oxford, 2010.

Smith, Barry: On Drawing Lines on a Map. In: A. U. Frank, W. Kuhn, D. M. Mark (Hg.): Spatial Information Theory. Proceedings of COSIT '95. Berlin, 1995, 475-484.

Vorländer, Hans, G. Melville: Geltungsgeschichten und Institutionsgeltung. Einleitende Aspekte. In: G. Melville, H. Vorländer (Hg.): Geltungsgeschichten. Über die Stabilisierung und Legitimierung institutioneller Ordnungen. IX-XV, Köln, Weimar, Wien, 2002.

Wasserman, Ryan: The Constitution Question. In: Nous 38. 2004, 693-710. Weber, Max: Über einige Kategorien der verstehenden Soziologie. Zuerst in: Logos 4, 1913; ND in: M. Weber: Gesammelte Aufsätze zur Wissenschaftslehre. 7. Aufl., Tübingen: Mohr-Siebeck 1988, 427-474.

Weber, Max: Soziologische Grundbegriffe. In: M. Weber: Wirtschaft und Gesellschaft. Grundriß der verstehenden Soziologie. Hg. v. J. Winckelmann, 5. rev. Aufl., Studienausgabe, Tübingen, 1980 (1921), $1-30$.

Zaibert, Leo: Review of John Searle, The Construction of Social Reality. In: Grazer philosophische Studien 54, 1998, 230-233. 